\title{
Inter-Limb Coordinative Structure in a 200 m Front Crawl Event
}

\author{
Pedro Figueiredo ${ }^{1}$, João Paulo Vilas-Boas ${ }^{1}$, Ludovic Seifert ${ }^{2}$, Didier Chollet $^{2}$ and \\ Ricardo J. Fernandes, ${ }^{1, *}$ \\ ${ }^{1}$ University of Porto, Faculty of Sport, Cifi2d, Porto, Portugal \\ ${ }^{2}$ University of Rouen, Faculty of Sport Sciences, Rouen, France
}

\begin{abstract}
The Index of Coordination has been currently used as a tool that measures the inter-arm lag time between propulsive phases in front crawl swimming. The aim of the present study was to assess the inter-arm coordinative structure during a $200 \mathrm{~m}$ front crawl maximal effort, as well as to understand its interplay with the stroking parameters. One complete arm stroke cycle, without breathing, was digitized and analysed the relative duration of the different phases and identified the model of arm coordination by using the Index of Coordination in each length of $50 \mathrm{~m}$ of the $200 \mathrm{~m}$ front crawl. A one way repeated measures ANOVA, with Tukey post-hoc test was used to compare all the variables, and Pearson correlation coefficients were computed between Index of Coordination and the biomechanical (velocity, stroke rate, stroke length) and coordinative parameters $(p<0.05)$. Results showed an increase in the relative duration of the propulsive phases, which induced an increase in the Index of Coordination as fatigue developed in relation to a decrease in velocity, stroke rate and stroke length. Additionally, Index of Coordination was inversely related with entry/catch $(\mathrm{r}=-0.78)$ and the non-propulsive phases $(\mathrm{r}=-0.97)$, and positive related with the pull $(\mathrm{r}=0.54)$, push $(0.78)$ and propulsive phases $(r=0.97)$. The results highlighted that, in fatigue stages, swimmers tend to increase their propulsive continuity, corresponding to a reduction of the non-propulsive lag time between the two arms' propulsive actions.
\end{abstract}

Keywords: Arm coordination, biomechanics, fatigue, front crawl, performance, swimming.

\section{INTRODUCTION}

Technical performance in swimming has traditionally been evaluated through changes in velocity, stroke rate and stroke length. However, this approach does not provide specific information on the effectiveness of motor organization. Coordination emerge as a consequence of constrains imposed on action, reflecting a propensity towards selforganizing optimality in biological systems [1]. In the front crawl technique, the two arms move rhythmically in anti-phase inter-limb relationship, which reflects an intrinsic coordination mode [2], being one of the most important factors contributing to the generation of propulsive forces [3].

To assess the coordinative parameters in front crawl, Chollet et al. [4] proposed the Index of Coordination (IdC), a tool that measures the inter-arm lag time between propulsive phases. Following these authors, the three major patterns of arm coordination are the catch up, the superposition and the opposition modes, being the coordination employed by the swimmer determined by the relative contributions of each phase (entry/catch, pull, push and recovery) to the total duration of the arm stroke cycle.

The aim of the present study is to assess the IdC during a $200 \mathrm{~m}$ front crawl maximal effort, as well as to understand its interplay with the stroking parameters. The relative contri-

*Address correspondence to this author at the Faculty of Sport, Porto University, Portugal; Tel: +351 225074763; E-mail: Ricfer@ fade.up.pt bution of the arm stroke phases will also be assessed and discussed.

\section{METHODS}

Six male competitive swimmers volunteered to participate in this study $(20.3 \pm 2.8$ years old, $69.4 \pm 4.8 \mathrm{~kg}$, $177.2 \pm 5.9 \mathrm{~cm}, 183.3 \pm 7.0 \mathrm{~cm}$ of arm span and $10.0 \pm 2.6 \%$ of fat mass). All participants signed a written informed consent in which the experimental protocol was described.

Swimmers were monitored in a $200 \mathrm{~m}$ front crawl maximal test, when passing through a specific pre-calibrated space (calibration frame with dimensions of $3 \times 3 \times 3 \mathrm{~m}$ for the horizontal, vertical and lateral directions). Thirty points of calibration were used and the synchronisation of the images was obtained using a pair of lights visible in the field of view of each video camera.

Six stationary video cameras were used, being two above and four underwater (Sony ${ }^{\circledR}$ DCR-HC42E). One complete arm stroke cycle, without breathing, was analysed for each length of $50 \mathrm{~m}$, being assumed a six-beat kick synchronization. Test session took place in a $25 \mathrm{~m}$ indoor pool, being used push starts in order to minimize the starting effect.

The video images were digitized with APASystem (Ariel Dynamics, USA) at a frequency of $50 \mathrm{~Hz}$, manually and frame by frame in order to have a more objective analysis. Twenty one anatomical reference points were used. Image coordinates were transformed to 3D object-space coordinates using the DLT algorithm and then filtered using a low pass digital filter of $6 \mathrm{~Hz}$. Each movement was then divided in 
four distinct phases defined as follows [4]: (i) entry/catch, which corresponds to the time between the entry of the hand into the water and the beginning of its backward movement; (ii) pull, corresponding to the time between the beginning of the backward movement of the hand and its entry into the plane vertical to the shoulder (initial part of the propulsion); (iii) push, the time between the positioning of the hand below the shoulder and its exit from the water and (iv) recovery, corresponding to the time between the exit of the hand and its next entry into the water.

The duration of each phase was measured with a precision of $0.02 \mathrm{~s}$ and was expressed as a percentage of the duration of a complete stroke cycle. The duration of the propulsive and non-propulsive phases were the sum of pull and push, and of catch and exit phases, respectively.

The average horizontal velocity (v) was assessed by dividing the displacement of the centre of mass in one stroke cycle for its total duration. Stroke rate (SR) was assessed through the inverse of its time duration, being stroke length (SL) considered the horizontal displacement of the centre of mass during a stroke cycle.

\section{Statistical Analysis}

After the normality was checked, mean (SD) was computed for all variables. A one way repeated measures ANOVA, with Tukey post-hoc test was used to compare all the variables. Additionally, Pearson correlation coefficients were computed between IdC and the other biomechanical and coordinative parameters. The level of significance was set at $\mathrm{p}<0.05$.

\section{RESULTS}

In Table $\mathbf{1}$ it is possible to observe the mean (SD) values of the general biomechanical parameters (v, SR and SL) and of the coordinative organization parameters (IdC and stroke phases) for the four laps of the $200 \mathrm{~m}$ front crawl test.

In Table 2 there are presented the coefficients of correlation between the IdC and all the other biomechanical and arm stroke phase variables.

\section{DISCUSSION}

The purpose of this research was to study the behaviour of IdC through a $200 \mathrm{~m}$ maximal front crawl effort and its relationship with the general biomechanical parameters. Moreover, as differences in the visual determination of IdC depends on the operator expertise [5], the assessment was done through digitization.

IdC staid in the catch-up mode during the whole effort. In the fourth lap it was observed an IdC increase possible due to fatigue (as previously observed in $100 \mathrm{~m}$ race [6]). In this fatigue stages, swimmers tend to increase their propulsive continuity, as observed elsewhere [7]. However, it further reflected more time spent during the propulsive phase than greater force generation as the $\mathrm{v}$ and SL decreased in the last $50 \mathrm{~m}$ of the $200 \mathrm{~m}$. These facts could be explained by the development of local muscular fatigue [8], reflecting a declining capacity to deliver power output [9]. Nonetheless, no changes were noticed for the SR, being the swimmers unable to increase it in order to compensate the SL decrease as suggested before (eg. [10]). These facts lead to an increase of the relative duration of the propulsive phases, reflecting the inability to generate enough propulsive force, as a consequence temporal structure changed [6].

Additionally, in the non-propulsive phases, IdC only was related with the entry/catch phase, implying that the relative time duration between the entry and the beginning of the pull phase has a great negative influence on the coordination

Table 1. Mean (SD) Values of the Velocity, Stroke Rate, Stroke Length, Index of Coordination, Entry/Catch, Pull, Push, Recovery, Propulsive Phases and Non-Propulsive Phases in the four Laps of the $200 \mathrm{~m}$ front Crawl

\begin{tabular}{|c|c|c|c|c|c|c|c|c|c|c|}
\hline & $\begin{array}{l}\text { Velocity } \\
\left(\mathbf{m} .^{-1}\right)\end{array}$ & $\begin{array}{c}\text { Stroke Rate } \\
\left(\text { Stroke.min }^{-1}\right)\end{array}$ & $\begin{array}{l}\text { Stroke Length } \\
\left(\mathrm{m} . \text { Stroke }^{-1}\right)\end{array}$ & $\begin{array}{c}\text { Index of } \\
\text { Coordination }(\%)\end{array}$ & $\begin{array}{c}\text { Entry/ } \\
\text { Catch }(\%)\end{array}$ & Pull (\%) & Push (\%) & $\begin{array}{c}\text { Recovery } \\
(\%)\end{array}$ & $\begin{array}{c}\text { Propulsive } \\
(\%)\end{array}$ & $\begin{array}{c}\text { Non- } \\
\text { Propulsive (\%) }\end{array}$ \\
\hline $\begin{array}{c}1^{\mathrm{st}} \\
50 \mathrm{~m}\end{array}$ & $\begin{array}{c}1.64 \\
(0.04)\end{array}$ & 49.08 (3.04) & $2.01(0.15)$ & $-13.1(2.7)$ & $37.7(4.9)$ & $23.1(1.4)$ & $14.1(1.2)$ & $25.1(3.7)$ & $37.2(2.6)$ & $62.8(2.6)$ \\
\hline $\begin{array}{c}2^{\text {nd }} \\
50 \mathrm{~m}\end{array}$ & $\begin{array}{c}1.48 \\
(0.07)^{\mathrm{a}}\end{array}$ & $43.88(3.18)^{\mathrm{a}}$ & $2.03(0.11)$ & $-13.5(3.8)$ & $39.0(5.1)$ & $12.4(1.6)$ & $24.3(3.4)$ & $24.4(3.1)$ & $36.6(4.0)$ & $63.4(4.0)$ \\
\hline $\begin{array}{l}4^{\text {th }} \\
50 \mathrm{~m}\end{array}$ & $\begin{array}{c}1.40 \\
(0.08)^{\text {a,b,c }}\end{array}$ & $44.33(2.37)^{\mathrm{a}}$ & $1.89(0.15)^{\mathrm{b}}$ & $-11.7(3.9)$ & $37.8(5.2)$ & 13.5 (2.9) & $24.9(4.7)$ & $23.8(2.4)$ & $38.4(4.3)$ & $\underset{b, c}{61.6(4.3)}$ \\
\hline
\end{tabular}

${ }^{a, b, c}$ Significantly different from the first, second and third laps, respectively $(\mathrm{p}<0.05)$.

Table 2. Correlation's Matrix Obtained Between Index of Coordination and Velocity, Stroke Rate, Stroke Length, Entry/Catch Phase, Pull Phase, Push Phase, Recovery, Propulsive Phases and Non-Propulsive Phases

\begin{tabular}{|c|c|c|c|c|c|c|c|c|c|}
\hline & $\begin{array}{c}\text { Velocity } \\
\left(\mathrm{m} . \mathrm{s}^{-1}\right)\end{array}$ & $\begin{array}{c}\text { Stroke Rate } \\
\left(\text { Stroke.min }{ }^{-1}\right)\end{array}$ & $\begin{array}{l}\text { Stroke Length } \\
\left(\mathbf{m} . S^{-S t r o k e} \mathbf{k}^{-1}\right)\end{array}$ & $\begin{array}{c}\text { Entry/ } \\
\text { Catch (\%) }\end{array}$ & Pull (\%) & Push (\%) & $\begin{array}{c}\text { Recovery } \\
(\%)\end{array}$ & $\begin{array}{c}\text { Propulsive } \\
(\%)\end{array}$ & $\begin{array}{c}\text { Non-Propulsive } \\
(\%)\end{array}$ \\
\hline $\begin{array}{c}\text { Index of } \\
\text { coordination }(\%)\end{array}$ & ns & ns & $\mathrm{ns}$ & -0.78 & 0.54 & 0.78 & ns & 0.97 & -0.97 \\
\hline
\end{tabular}


mode, which should be diminished specially under fatigue conditions.

\section{ACKNOWLEDGMENT}

This study was supported by grants: SFRH / BD / 38462 / 2007 and PTDC/DES/101224/2008.

\section{REFERENCES}

[1] Newel K. Constrains on the development of coordination. In: Wade $\mathrm{M}$, Whiting $\mathrm{H}$, Eds. Motor development in children: aspect of coordination and control. Dordrecht: Martinus Nijhoff Publishers 1986; pp. 341-60.

[2] Nikodelis T, Kollias I, Hatzitaki V. Bilateral inter-arm coordination in freestyle swimming: Effect of skill level and swimming speed. 2005; 23: 737-45

[3] Potdevin F, Bril B, Sidney M, Pelayo P. Stroke frequency and arm coordination in front crawl swimming. Int J Sports Med 2006; 27 : 193-8.
[4] Chollet D, Chalies S, Chatard JC. A new index of coordination for the crawl: description and usefulness. Int J Sports Med 2000; 21(1): 54-9.

[5] Seifert L, Schnitzler C, Aujouannet YA, Carter M, Rouard AH, Chollet D. Comparison of subjective and objective methods of determination of stroke phases to analyse arm coordination in frontcrawl. Port J Sports Sci 2006; 6 (2): 92-4.

[6] Seifert L, Chollet D, Chatard JC. Kinematic changes during a 100 $\mathrm{m}$ front crawl: effects of performance level and gender. Med Sci Sports Exerc 2007; 39 (10): 1784-93.

[7] Alberty M, Sidney M, Huot-Marchand F, Hespel JM, Pelayo P. Intracyclic velocity variations and arm coordination during exhaustive exercise in front crawl stroke. Int J Sports Med 2005; 26: 471-5.

[8] Keskinen K, Komi P. Stroking characteristics of front craw swimming during exercice. J Appl Biomech 1993; 9: 219-26.

[9] Toussaint HM, Carol A, Kranenborg H, Truijens M. Effect of fatigue on stroking characteristics in an arms-only 100-m frontcrawl race. Med Sci Sports Exerc 2006; 38: 1635-42.

[10] Coelho J, Fernandes R, Colaço C, Soares S, Vilas-Boas J. Kinetics of glycolysis during the short-course 100-m crawl swimming event. J Sports Sci 2008; 26(1): 5.

(C) Figueiredo et al.; Licensee Bentham Open

This is an open access article licensed under the terms of the Creative Commons Attribution Non-Commercial License.

(http://creativecommons.org/licenses/by-nc/3.0/) which permits unrestricted, non-commercial use, distribution and reproduction in any medium, provided the work is properly cited. 